\title{
Verapamil Inhibits Aspergillus Biofilm, but Antagonizes Voriconazole
}

\author{
Hasan Nazik ${ }^{1,2, *, \dagger}$, Varun Choudhary ${ }^{1}$ and David A. Stevens ${ }^{1,2}$ \\ 1 California Institute for Medical Research, 2260 Clove Dr., San Jose, CA 95128, USA; \\ varun746@gmail.com (V.C.); stevens@stanford.edu (D.A.S.) \\ 2 Division of Infectious Diseases and Geographic Medicine, Stanford University Medical School, \\ Stanford, CA 94305, USA \\ * Correspondence: hasannazik01@gmail.com; Tel.: +1-408-998-4554; Fax: +1-408-998-2723 \\ + Permanent address: Department of Medical Microbiology, Faculty of Medicine, Istanbul University, \\ Istanbul 34452, Turkey.
}

Received: 10 August 2017; Accepted: 18 September 2017; Published: 20 September 2017

\begin{abstract}
The paucity of effective antifungals against Aspergillus and increasing resistance, the recognition of the importance of Aspergillus biofilm in several clinical settings, and reports of verapamil—a calcium channel blocker-efficacy against Candida biofilm and hyphal growth, and synergy with an azole antifungal in vitro, led to a study of verapamil \pm voriconazole against Aspergillus. Broth macrodilution methodology was utilized for MIC (minimum inhibitory concentration) and MFC (minimum fungicidal concentration) determination. The metabolic effects (assessed by XTT [2,3-bis[2-methoxy-4-nitro-5-sulfophenyl]-2H-tetrazolium-5-carboxanilide inner salt]) on biofilm formation by conidia were studied upon exposure to verapamil, verapamil plus voriconazole, or voriconazole alone. For biofilm formation, we found less inhibition from the combinations than with either drug alone, or less inhibition from the combination than that of the more potent drug alone. For preformed biofilm, we found no significant change in activity comparing voriconazole alone compared to added verapamil, and no significant alteration of activity of the more potent voriconazole, at any concentration in the range tested, by addition of a concentration of verapamil that is inhibitory alone. In full checkerboard assays with planktonic fungus, there was no indication of any effect of one drug on the other (indifference). Although verapamil was similarly inactive against planktonic Aspergillus, as with Candida, verapamil was indeed active against Aspergillus biofilm. However, indifference and antagonism was found with voriconazole.
\end{abstract}

Keywords: verapamil; Aspergillus; biofilm; voriconazole

\section{Introduction}

In eukaryotes, the calcium signaling pathway plays an essential role in response to cellular stress, including oxidative stress. On perception of stress by the cell, there is a calcium influx, leading to the upregulation of calcium-dependent genes that are required for the response to stress. Verapamil is a calcium channel blocker. Its effect is to decrease calcium fluctuation and thus the calcium influx, decreasing the oxidative stress response.

In Candida albicans, the depression of the calcium flux by verapamil results in increased sensitivity to oxidative stress, increased reactive oxygen species, and mitochondrial dysfunction, with a net result of fungal inhibition [1]. In addition, many fungi maintain a cytoplasmic calcium gradient, with high calcium concentrations at the growing tip, a necessary condition for polarized growth [2]. Thus in C. albicans, the calcium signaling pathway is necessary for morphogenetic transformation to the hyphal state, the more invasive state of the organism. Blockage of the calcium flux by verapamil inhibits hyphal development [3]. In addition, candidal adherence and the ability to colonize the 
mammalian gut are decreased by verapamil treatment. Calcium homeostasis is also necessary for biofilm development [4]. Verapamil has activity in vitro against the formation and maintenance of candidal biofilm, and acts synergistically with antifungals against candidal biofilm formation [5].

Although a number of effective antifungal drugs have been developed against $C$. albicans, the antifungal armamentarium against Aspergillus is more limited [6], and resistance development to the agent of choice—voriconazole [7]—is increasing [8]. Aspergillus biofilm formation is a problem in several clinical situations, particularly chronic lung infections, such as in cystic fibrosis $[9,10]$. Verapamil has a well-studied pharmacology and toxicology [11]. We therefore investigated whether verapamil, alone or in combination, could offer something promising against Aspergillus biofilm.

\section{Materials and Methods}

For reagents, Verapamil was obtained from Hospira (Lake Forest, IL, USA) and Sigma (St. Louis, MO, USA) and compared in the assays of inhibition to be described. The potency from these two sources was found to be equivalent, in saline or in RPMI1640 medium, and they were then used interchangeably. We also noted no differences if verapamil stocks were first constituted in distilled water or dimethyl sulfoxide (DMSO). Voriconazole was obtained from Pfizer, New York City, and stock was made in DMSO for further dilution down to test conditions with RPMI. A control of the same DMSO concentration without voriconazole verified no effect of DMSO alone. Large batches of the reagents were prepared in aliquots and frozen, and a fresh aliquot used in each experiment.

For Aspergillus biofilm inhibition assays, Aspergillus fumigatus (strain AF10, a virulent clinical isolate) (ATCC ${ }^{\circledR}$ 90240 ${ }^{\mathrm{TM}}$ ) [12] biofilm formation and preformed biofilm were prepared as previously detailed [13]. The presence of biofilm was verified: hyphal mats with biofilm form [9] were visualized by optical microscopy, showing the same arrangement as has been noted in optical, confocal and electron microscopy [13,14]. A 96-well plate assay [15] was used, with RPMI1640 medium, 8 replicate wells/each experimental group, preparation of the Af target from conidiating cultures (on potato dextrose agar) (PDA), and XTT (2,3-bis[2-methoxy-4-nitro-5-sulfophenyl]-2H-tetrazolium-5-carboxanilide inner salt) (Sigma) readout of Af inhibition, as previously described [13,15]. In brief, for studies of biofilm formation, $2 \times 10^{3}$ conidia/well was co-cultured with reagents for $16 \mathrm{~h}$ at $37^{\circ} \mathrm{C}$, shaking $65-70 \mathrm{RPM}$. At $16 \mathrm{~h}$, a biofilm had formed, as noted previously [13]. For study of preformed Aspergillus biofilm, reagents were added only after $16 \mathrm{~h}$, then incubated for $24 \mathrm{~h}$. After $40 \mathrm{~h}$, the metabolic activity of the biofilm was measured with XTT assay. XTT results of each experimental group were compared to those of a concurrent Aspergillus control (no added reagents), by $t$-test. Biofilm formation was studied in six experiments, and preformed biofilm in nine experiments.

For MIC and MFC determination, two additional Af clinical isolates, CIMR nos. 15-31 and 15-37 were also studied. Broth macrodilution methodology was utilized as described [16], including concurrent testing of a known voriconazole-susceptible isolate as a control for drug batch activity. Minimum fungicidal concentrations (MFCs) were determined by subculture of the drug-treated tubes, with an MFC endpoint of $\geq 96 \%$ killing of original inoculum [17].

Data from experiments using XTT were analyzed using one-way ANOVA with a Tukey's post-test for multiple comparisons and Student's $t$-test was used if two groups were compared. All statistical analyses were performed using Graph-Pad Prism (GraphPad Software, version 3, La Jolla, CA, USA). Statistical significance was considered $p<0.05$.

\section{Results}

The initial studies were performed to examine the dose response for both reagents, in two- and four-fold dilution ranges. For biofilm formation, concentrations of verapamil 2500 to $39 \mathrm{mcg} / \mathrm{mL}$ showed significant $(p<0.01-0.001)$ inhibition compared to the no drug control. The lowest concentration of verapamil that gave repeated statistically significant inhibitory results were $7.8 \mathrm{mcg} / \mathrm{mL}$ for preformed biofilm, and $39 \mathrm{mcg} / \mathrm{mL}$ for assays of biofilm formation. For voriconazole, a range of $0.125-2 \mathrm{mcg} / \mathrm{mL}$ gave consistent inhibition for both preformed biofilm and biofilm formation, and this 
was satisfactory for our purposes, i.e., to provide a range from which concentrations could be selected for combination studies, allowing sufficient 'room' to demonstrate in the combination tubes either enhancement (additive or synergy response) or loss of activity (antagonism) compared to the single drug tube. For studies of drug interactions, it was desirable to use a concentration of one that gave inhibition compared to the control in the mid-range-i.e., approximately $50 \%$-and then examine the effect of a dilution series of the other drug. This is because the laboriousness, and time needed, in preparing the wells with biofilms and executing the experiments precludes doing a full checkerboard assay concurrently (as is possible in MIC assays). Thus it is desirable to have a drug level of the test drugs singly in the mid-range so as to be able to see, and test statistically, greater inhibition in the combination (indicating an additive effect or synergy) than one drug alone (i.e., a value below that of the one drug alone), or less inhibition (indicating antagonism, a value of the combination above that of the one drug alone), if either is present. We tested several concentrations of the single drugs in these assays to give us these desired conditions. Representative experiments are shown in Figures 1 and 2.

In Figure 1 (biofilm formation) we see the less inhibition by the combinations than that with either drug alone, or less inhibition from the combination than that of the more potent drug alone. These are defined as antagonism.

For preformed biofilm a dose-response for verapamil alone is shown in Figure 2. In Figure 2 (preformed biofilm) we see no significant change in activity comparing voriconazole alone vs. added verapamil (Figure 2A), and no significant alteration of activity of the more potent voriconazole, at any concentration in the range tested, by addition of a concentration of verapamil that is inhibitory alone (Figure 2B), both results shown in Figure 2 defined as indifference.

A

Effect of voriconazole $(\mu \mathrm{g} / \mathrm{ml})$ on inhibition of Aspergillus biofilm formation by verapamil $(\mu \mathrm{g} / \mathrm{ml})$

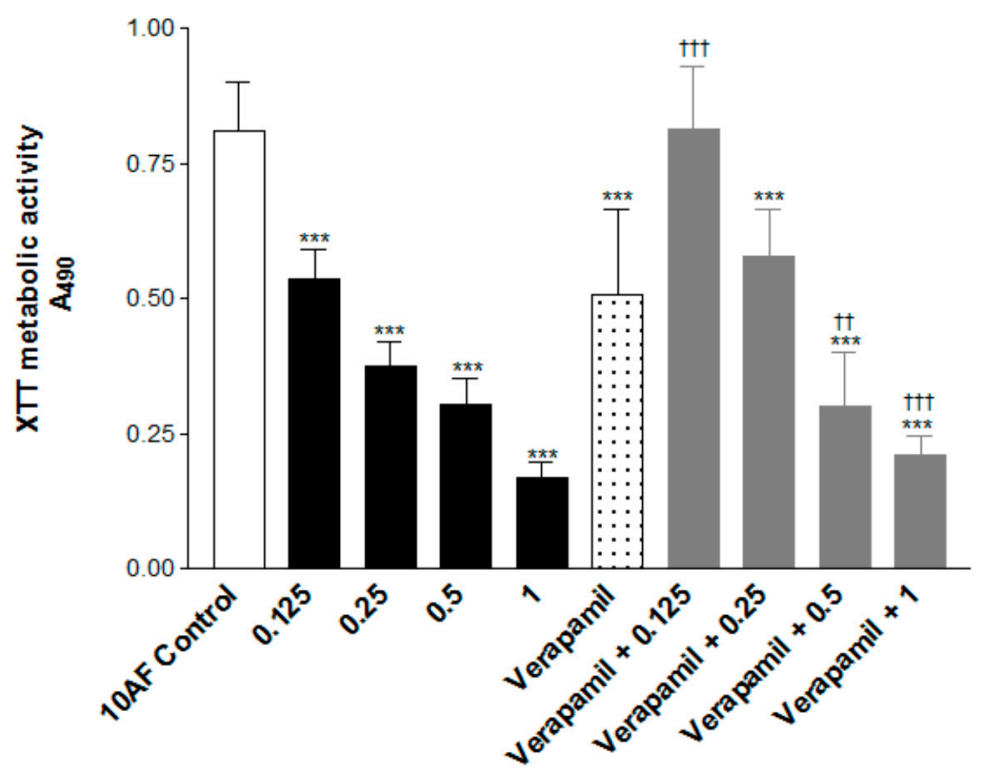

Figure 1. Cont. 
B

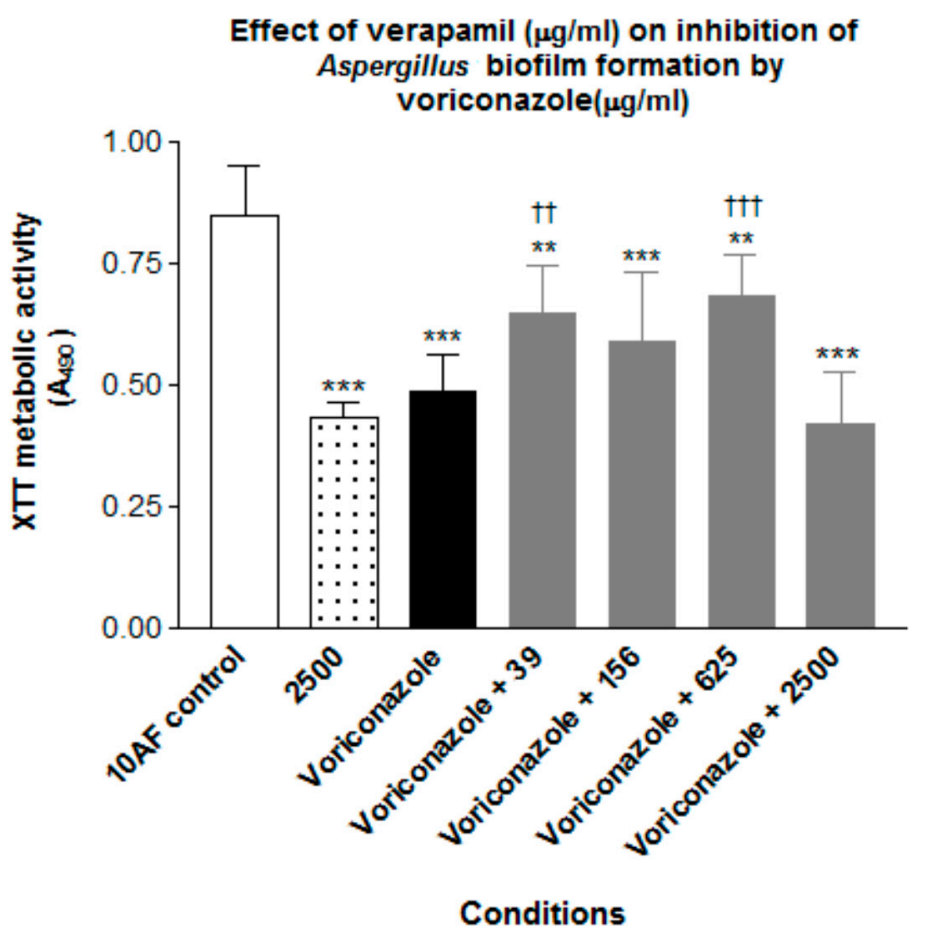

Figure 1. (A) Verapamil $39 \mathrm{mcg} / \mathrm{mL}$ alone was inhibitory, as shown here (sixth bar from left). This is a study of the effect of voriconazole doses on the effect of verapamil on formation of Aspergillus biofilm. The numbers 0.125 to 1 are final voriconazole concentrations in $\mathrm{mcg} / \mathrm{mL}$, alone (second through fifth bars from the left) or in combination with verapamil $39 \mathrm{mcg} / \mathrm{mL}$ (four right bars). ${ }^{* *}, p<0.001$ lower than the no drug control, left bar. ${ }^{+\dagger}$ and ${ }^{++\dagger}$ are $p<0.01$ and 0.001 , respectively, vs. verapamil alone. Verapamil +0.125 voriconazole is not only less inhibitory than verapamil alone $\left({ }^{++\dagger}\right)$, but also than 0.125 voriconazole alone $(p<0.001)$. Verapamil +0.25 voriconazole is not only less inhibitory than verapamil alone, but also less inhibitory than 0.25 voriconazole alone $(p<0.01)$. Verapamil $+1 \mathrm{mcg} / \mathrm{mL}$ voriconazole is not only less inhibitory than verapamil alone $\left({ }^{++\dagger}\right)$, but also less inhibitory than $1 \mathrm{mcg} / \mathrm{mL}$ voriconazole alone $(p<0.05)$. (B) The effect of verapamil doses on the effect of voriconazole on formation of Aspergillus biofilm can be seen. The numbers 39 to 2500 represent the final concentration of verapamil, alone or in combination, in $\mathrm{mcg} / \mathrm{mL}$. The final voriconazole concentration, alone or in combination was $0.125 \mathrm{mcg} / \mathrm{mL}{ }^{* *}$ and ${ }^{* * *}, p<0.01$ and 0.001 , respectively, compared to no drug control (left bar). ${ }^{+\dagger}$ and ${ }^{++\dagger}, p<0.01$ and 0.001 , respectively, less inhibition in combinations compared to voriconazole alone. Antagonism is again demonstrated.

A full checkerboard assay was performed using three strains in planktonic growth (data not illustrated). A range of voriconazole susceptibilities was included. In these studies the MIC of voriconazole alone was $0.5,0.25$, and $0.125 \mathrm{mcg} / \mathrm{mL}$ for strains $10 \mathrm{AF}, 15-77$ and 15-31, respectively, if a 50\% inhibition endpoint was used for the $\mathrm{MIC}$, and 4, 1, and $1 \mathrm{mcg} / \mathrm{mL}$, respectively, if a $100 \%$ endpoint (clear tube) was used. The MFCs were $>8,1$ and $1 \mathrm{mcg} / \mathrm{mL}$, respectively. The MIC and MFC of verapamil was $>624 \mathrm{mcg} / \mathrm{mL}$ with all isolates and either endpoint. There was no indication of any effect of one drug on the other (indifference). 


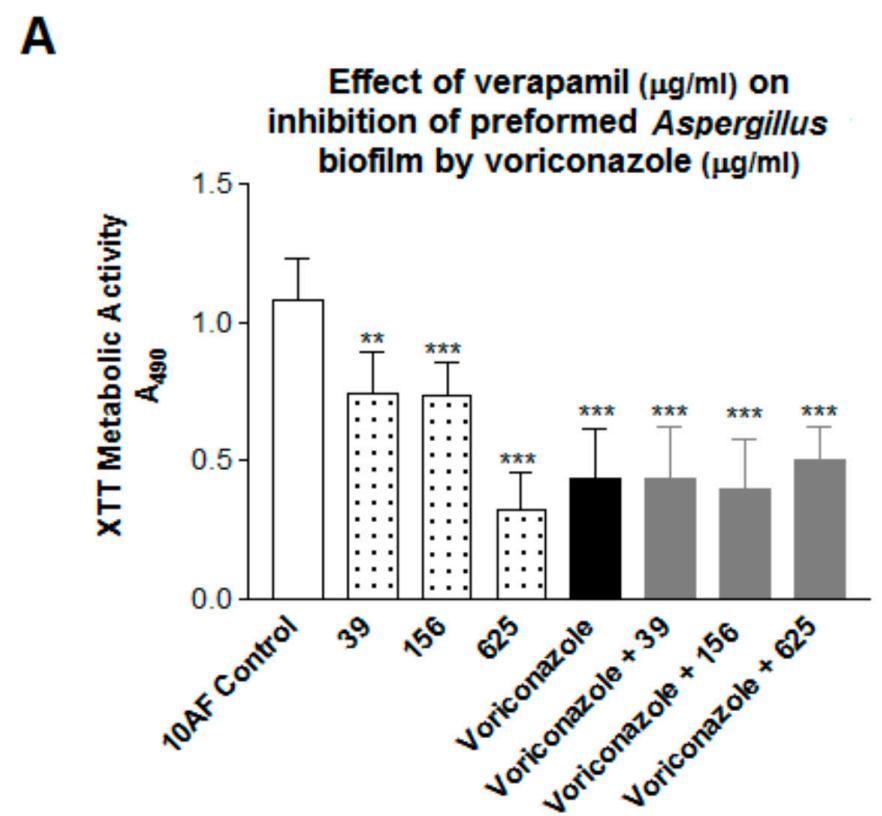

B

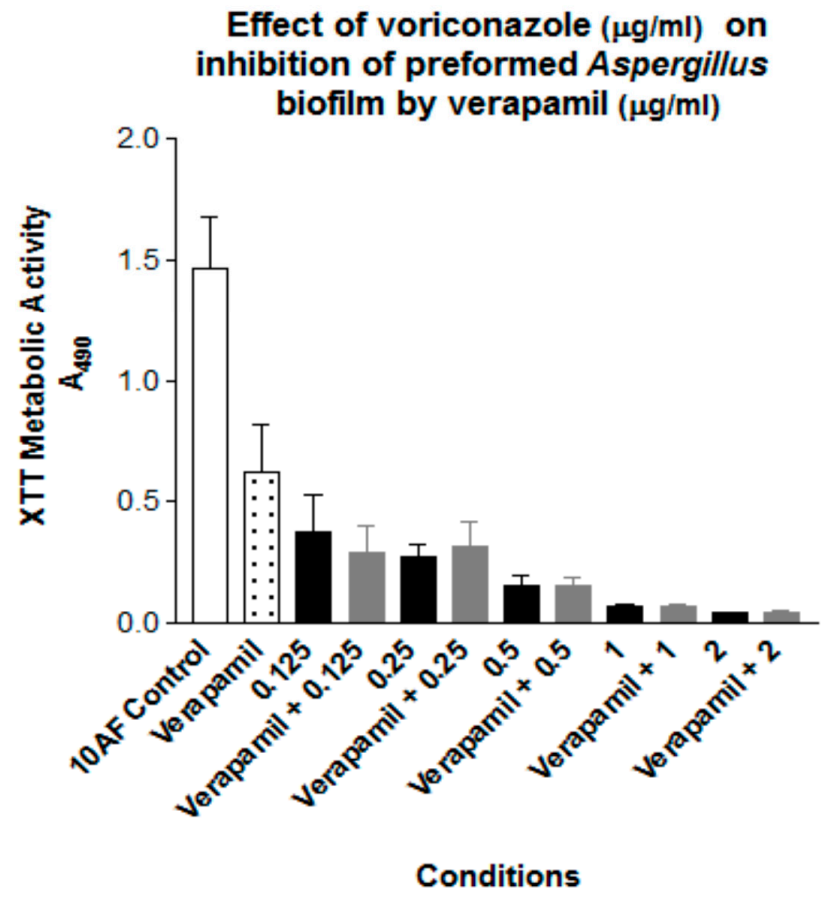

Figure 2. (A) Effect of verapamil doses on the effect of voriconazole on preformed Af biofilm. The voriconazole dose tested was $0.125 \mathrm{mcg} / \mathrm{mL}$ final concentration (4 right bars). The numbers $39,156,625$ refer to verapamil final concentrations in $\mathrm{mcg} / \mathrm{mL}$. Two and three asterisks refer to $p<0.01$ and 0.001, respectively, compared to no-drug control (left bar). The combination bars are not different from voriconazole alone; no potentiation of voriconazole by verapamil. (B) Effect of voriconazole doses on the effect of verapamil on preformed Af biofilm. The verapamil concentration tested, alone (second bar from left) or in combination, was $39 \mathrm{mcg} / \mathrm{mL}$. The numbers 0.125 through 2 are final concentrations of voriconazole in $\mathrm{mcg} / \mathrm{mL}$, alone or in combination. The no-drug control is at the left, and is $p<0.001$ different from all other bars. The voriconazole alone bars are plotted here side-by-side with the corresponding combination bars; there are no differences in any pair. Indifference is again demonstrated. 


\section{Discussion}

The lack of verapamil activity against $A$. fumigatus planktonic growth is consistent with the report of inactivity against C. albicans planktonic growth (inactivity at $320 \mathrm{mcg} / \mathrm{mL}$, different medium) [1]. The concentrations in our study that inhibited Aspergillus biofilm formation approximate those reported to inhibit C. albicans biofilm formation $(20 \mathrm{mcg} / \mathrm{mL})$ [5], a concentration that also inhibited the metabolism of preformed C. albicans biofilm, and approximated what we found with preformed Aspergillus biofilm.

There were some unexpected findings from our studies. First, we could not show the synergy of verapamil with an azole against Aspergillus biofilm, either its formation or on preformed biofilm-as has, in contrast, been reported with an azole and C. albicans biofilm [5] -and our interaction was in part a negative one. The reason for those differences is a subject for future research. It will be important in such studies to examine the applicability of our findings to other, clinical, A. fumigatus isolates, and for our findings to be confirmed by other investigators.

Second, although it has been generally held that Aspergillus biofilm is resistant to voriconazole [18-20], that was not our finding. Those differences could relate to differences in medium used, differences in A. fumigatus strains, and/or differences in assay methods, and endpoint determinations. It has also been reported [18] that preformed Aspergillus biofilm is more drug-resistant than biofilm formation, whereas we did not find this to be the case with either drug studied, although the reference cited was not addressing a drug such as verapamil, our verapamil concentrations inhibitory to biofilm formation and preformed biofilm were not distantly disparate, and our voriconazole concentrations were not titered below $0.125 \mathrm{mcg} / \mathrm{mL}$ (which then possibly could have shown differences).

The relevant serum concentrations of verapamil in its cardiologic use are $0.1-0.4 \mathrm{mcg} / \mathrm{mL}$ [11]. This would suggest that verapamil itself would not be promising for therapy either against planktonic A. fumigatus or C. albicans, their biofilms, or in combination with azoles, given the verapamil endpoints derived in our studies. Verapamil is also an inhibitor of the p-glycoprotein drug efflux pump [21], a property that could also affect these drug combination studies against fungi, but this property might also affect penetration of other (e.g., antifungal) drugs into tissues. However, our findings and these cautions do not preclude the possible utility of other calcium channel blockers as adjuncts to antifungal therapy, particularly against biofilm homeostasis.

Acknowledgments: Funded in part by a gift from John Flatley. We thank Gabriele Sass and Marife Martinez for their contribution to some assays.

Author Contributions: Hasan Nazik and David A. Stevens conceived and designed the experiments; Hasan Nazik and Varun Choudhary performed the experiments; Hasan Nazik, Varun Choudhary, and David A. Stevens analyzed the data; Hasan Nazik and David A. Stevens wrote the paper.

Conflicts of Interest: The authors declare no conflict of interest.

\section{References}

1. Yu, Q.; Xiao, C.; Zhang, K.; Jia, C.; Ding, X.; Zhang, B.; Wang, Y.; Li, M. The calcium channel blocker verapamil inhibits oxidative stress response in Candida albicans. Mycopathologia 2014, 177, 167-177. [CrossRef] [PubMed]

2. Brand, A.; Shanks, S.; Duncan, V.M.S.; Yang, M.; Mackenzie, K.; Gow, N.A. Hyphal orientation of Candida albicans is regulated by a calcium-dependent mechanism. Curr. Biol. 2007, 17, 347-352. [CrossRef] [PubMed]

3. Yu, Q.; Ding, X.; Zhang, B.; Xu, N.; Jia, C.; Mao, J.; Zhang, B.; Xing, L.; Li, M. Inhibitory effect of verapamil on Candida albicans hyphal development, adhesion and gastrointestinal colonization. FEMS Yeast Res. 2014, 14, 633-641. [CrossRef] [PubMed]

4. Ramage, G.; Wickes, B.L.; López-Ribot, J.L. Inhibition on Candida albicans biofilm formation using divalent cation chelators (EDTA). Mycopathologia 2007, 164, 301-306. [CrossRef] [PubMed] 
5. Yu, Q.; Ding, X.; Xu, N.; Cheng, X.; Qian, K.; Zhang, B.; Xing, L.; Li, M. In vitro activity of verapamil alone and in combination with fluconazole or tunicamycin against Candida albicans biofilms. Int. J. Antimicrob. Agents 2013, 41, 179-182. [CrossRef] [PubMed]

6. Rex, J.; Stevens, D.A. Drugs active against fungi, Pneumocystis and Microsporidia. In Principles and Practice of Infectious, 8th ed.; Bennett, J., Dolin, R., Blaser, M., Eds.; Elsevier Publ.: Philadelphia, PA, USA, 2014.

7. Patterson, T.F.; Thompson, G.R.; Denning, D.W.; Fishman, J.A.; Hadley, S.; Herbrecht, R.; Kontoyiannis, D.P.; Marr, K.A.; Morrison, V.A.; Nguyen, M.H.; et al. Practice guidelines for the diagnosis and management of aspergillosis: 2016 update by the infectious diseases society of America. Clin. Infect. Dis. 2016, 63, 433-442. [CrossRef] [PubMed]

8. Denning, D.W.; Bowyer, P. Voriconazole resistance in Aspergillus fumigatus: Should we be concerned? Clin. Infect. Dis. 2013, 57, 521-523. [CrossRef] [PubMed]

9. Beauvais, A.; Latgé, J. Aspergillus biofilm in vitro and in vivo. Microbiol. Spectr. 2015, 3. [CrossRef] [PubMed]

10. Reichhardt, C.; Stevens, D.A. Cegelski L: Fungal biofilm composition and opportunities in drug discovery. Future Med. Chem. 2016, 8, 1455-1468. [CrossRef] [PubMed]

11. Melmon, K.; Morelli, H.; Hoffman, B.B.; Nierenberg, D.W. Melmon and Morrelli's Clinical Pharmacology: Basic Principles in Therapeutics, 3rd ed.; McGraw-Hill Inc.: New York, NY, USA, 1992.

12. Denning, D.W.; Stevens, D.A. Efficacy of cilofungin alone and in combination with amphotericin B in a murine model of disseminated aspergillosis. Antimicrob. Agents Chemother. 1991, 35, 1329-1333. [CrossRef] [PubMed]

13. Ferreira, J.A.G.; Penner, J.C.; Moss, R.B.; Haagensen, J.A.J.; Clemons, K.V.; Spormann, A.M.; Nazik, H.; Cohen, K.; Banaei, N.; Carolino, E.; et al. Inhibition of Aspergillus fumigatus and its biofilm by Pseudomonas aeruginosa is dependent on the source, phenotype and growth conditions of the bacterium. PLoS ONE 2015, 10, e0134692. [CrossRef] [PubMed]

14. Reichhardt, C.; Ferreira, J.A.; Joubert, L.M.; Clemons, K.V.; Stevens, D.A.; Cegelski, L. Analysis of the Aspergillus fumigatus biofilm extracellular matrix by solid-state nuclear magnetic resonance spectroscopy. Eukaryot. Cell 2015, 14, 1064-1072. [CrossRef] [PubMed]

15. Nazik, H.; Penner, J.C.; Ferreira, J.A.; Haagensen, J.A.J.; Cohen, K.; Spormann, A.M.; Martinez, M.; Chen, V.; Hsu, J.L.; Clemons, K.V.; et al. Effects of iron chelators on the formation and development of Aspergillus fumigatus biofilm. Antimicrob. Agents Chemother. 2015, 59, 6514-6520. [CrossRef] [PubMed]

16. Clinical and Laboratory Standards Institute. Reference Method for Broth Dilution Antifungal Susceptibility Testing of Filamentous Fungi, 2nd ed.; approved standard. CLSI document M38-A2; Clinical and Laboratory Standards Institute: Wayne, PA, USA, 2008.

17. Stevens, D.A.; Aristizibal, B.H. In vitro antifungal activity of novel azole derivatives with a morpholine ring, UR-9746 and UR-9751, and comparison with fluconazole. Diagn. Microbiol. Infect. Dis. 1997, 29, 103-106. [CrossRef]

18. Mowat, E.; Lang, S.; Williams, C.; McCulloch, E.; Jones, B.; Ramage, G. Phase-dependent antifungal activity against Aspergillus fumigatus developing multicellular filamentous biofilms. J. Antimicrob. Chemother. 2008, 62, 1281-1284. [CrossRef] [PubMed]

19. Rajendran, R.; Mowat, E.; McCulloch, E.; Lappin, D.F.; Jones, B.; Lang, S.; Majithiya, J.B.; Warn, P.; Williams, C.; Ramage, G. Azole resistance of Aspergillus fumigatus biofilms is partly associated with efflux pump activity. Antimicrob. Agents Chemother. 2011, 55, 2092-2097. [CrossRef] [PubMed]

20. Seidler, M.J.; Salvenmoser, S.; Müller, F.M.C. Aspergillus fumigatus forms biofilms with reduced antifungal drug susceptibility on bronchial epithelial cells. Antimicrob. Agents Chemother. 2008, 52, 4130-4136. [CrossRef] [PubMed]

21. Bansal, T.; Mishra, G.; Jaggi, M.; Khar, R.K.; Talegaonkar, S. Effect of P-glycoprotein inhibitor, verapamil, on oral bioavailability and pharmacokinetics of irinotecan in rats. Eur. J. Pharm. Sci. 2009, 36, 580-590. [CrossRef] [PubMed]

(C) 2017 by the authors. Licensee MDPI, Basel, Switzerland. This article is an open access article distributed under the terms and conditions of the Creative Commons Attribution (CC BY) license (http:/ / creativecommons.org/licenses/by/4.0/). 